\title{
Clinical outcomes of direct-acting antiviral therapy in patients with compensated hepatitis $\mathrm{C}$ virus-related cirrhosis
}

\author{
Estefania Berge, Ana Arencibia, Elena Otón, Luis Cejas, Silvia Acosta, Francisco Pérez \\ Liver Unit, Hospital Universitario Nuestra Señora de la Candelaria, 38010 Santa Cruz de Tenerife, Spain.
}

Correspondence to: Dr. Estefanía Berge, Liver Unit, Hospital Universitario Nuestra Señora de la Candelaria, Carretera General del Rosario, 145 , 38010 Santa Cruz de Tenerife, Spain. E-mail: estefania.berge@gmail.com

How to cite this article: Berge E, Arencibia A, Otón E, Cejas L, Acosta S, Pérez F. Clinical outcomes of direct acting antiviral therapy in patients with compensated hepatitis C virus-related cirrhosis. Hepatoma Res 2017;3:209-14.

\section{Article history: \\ Received: 25 Jun 2017 \\ Accepted: 12 Sep 2017 \\ Published: 27 Sep 2017}

Key words:

Direct-acting antiviral therapy, compensated cirrhosis, hepatocellular carcinoma, clinical decompensation

\begin{abstract}
Aim: The aim was to assess the clinical impact of direct-acting antiviral treatment in patients with compensated hepatitis $\mathrm{C}$ virus-related cirrhosis after one year of follow-up. Methods: An observational retrospective study was conducted on 129 consecutive patients with compensated cirrhosis treated in 2015, analyzing the evolution of liver function and the development of hepatocellular carcinoma and clinical decompensations. Results: The median follow-up time was 16 months. Most patients were males (73\%), the mean age was 58.1 years and the most frequent genotype was $1 \mathrm{~b}$ (52.2\%). All participants were Child-Pugh A class at the start of the treatment and the median model for end-stage liver disease (MELD) score was 7. Four patients (4.4\%) suffered a decompensation: three episodes of ascites and one acute on chronic liver failure. The incidence of de novo hepatocellular carcinoma during the follow-up was $3.6 \%$. Seven patients (7.8\%) improved MELD score more than one point and in 11 patients $(12.2 \%)$ it worsened more than one point. There was a significant improvement in the mean platelets count $[P<0.001,95 \%$ confidence interval (CI): -26,360, -12,096] and in the mean albumin levels $(P<0.001,95 \%$ CI: $-322,-130)$ after treatment. Conclusion: Direct-acting antiviral treatment is not associated in the short term with a decrease in the development of hepatic decompensation or hepatocellular carcinoma compared to what it was reported for untreated compensated cirrhotic patients. There is an improvement in pre and post-treatment platelet counts and albumin levels showing a probable improvement of the hepatic function.
\end{abstract}

\section{INTRODUCTION}

Chronic hepatitis C virus (HCV) infection affects around 160 million people worldwide ${ }^{[1]}$. Around $16 \%$ of patients will develop cirrhosis after 20 years of infection ${ }^{[2]}$, although fibrosis progression can vary due to several factors such as age, alcohol consumption or hepatitis B or human immunodeficiency virus (HIV) co-infection ${ }^{[3]}$. Once cirrhosis is established, a yearly incidence of hepatocellular carcinoma of $1.4-3.4 \% \%^{[4-6]}$, and a yearly incidence of hepatic decompensation (including episodes of ascites, jaundice, hepatic 
encephalopathy or variceal bleeding) of $3.9-5.7 \%[4,6]$ has been reported.

Before 2011, the best potentially curative treatment option for chronic HCV infection was pegylated interferon in combination with ribavirin. However, sustained virological response rates were reported to be as low as $33 \%$ in cirrhotic patients ${ }^{[7]}$, with an significant number of side effects ${ }^{[8]}$. However, it has been demonstrated that the achievement of a sustained virological response after interferon-based therapy is associated with lower rates of hepatocellular carcinoma and with lower rates of hepatic decompensation ${ }^{[9,10]}$. A regression of fibrosis after viral eradication has also been reported ${ }^{[11]}$.

The arrival of second-generation direct-acting antivirals improved the sustained virological response rates to more than $90 \%$, even in compensated cirrhotic patients, with fewer side effects ${ }^{[12,13]}$. However, the achievement of sustained virological response with this treatment does not appear to be associated with a decrease in the occurrence of hepatocellular carcinoma in the short term ${ }^{[14]}$. It may even be associated with a higher rate of tumor recurrence than what it is expected ${ }^{[15]}$. A recent prospective multicenter study did not find any evidence of an increased risk of hepatocellular carcinoma recurrence in patients treated with direct-acting antivirals ${ }^{[16]}$. One report additionally demonstrated an improvement in liver function tests among patients with decompensated liver disease after treatment with oral antiviral therapy ${ }^{[11]}$.

The aim of our study was to assess the clinical impact of direct-acting antiviral treatment in terms of the evolution of liver function and in terms of the development of clinical decompensations and hepatocellular carcinoma in patients with compensated HCV-related cirrhosis after one year of follow-up.

\section{METHODS}

Data from all the patients with compensated HCVrelated cirrhosis, without co-existent HIV or hepatitis $B$ infection, who were treated at our center with directacting antivirals between January and October 2015, were retrospectively collected. At the end of October 2016, the database included 129 patients. We excluded 39 patients because they did not complete a followup of 12 months, which included at least physical examination, hepatic ultrasound, and blood tests every 6 months. Fourteen patients were lost to follow-up during the first year and 25 patients had the last clinic or ultrasound appointment after October 2016.

The diagnosis of cirrhosis was previously established by biopsy, transient elastography (> $14.5 \mathrm{Kpa}$ ) or unequivocal clinical diagnosis (chronic HCV with previous episodes of decompensation or with imaging tests showing portal hypertension signs).

Sustained virological response 12 weeks posttreatment [sustained virological response 12 (SVR12)] was defined as undetectable HCV RNA at week 12 after the end of therapy. For HCV RNA detection we used real time polymerase chain reaction, with a limit of detection of $15 \mathrm{IU} / \mathrm{mL}$.

Follow-up started the first day of treatment, which was defined as time 0 . We analyzed: (1) the development of hepatocellular carcinoma. We performed an ultrasound every 6 months and, when it showed a suspicious focal lesion, the diagnosis of hepatocellular carcinoma was completed with a triple-phase computerized tomography scan and/or with a contrast enhanced magnetic resonance imaging; (2) the development of hepatic decompensation, which included jaundice, variceal bleeding, ascites and/or encephalopathy; (3) the evolution of liver function, using Child-Pugh and MELD scores, which were calculated on the first day of treatment and on the last clinic visit, at least one year later. We also performed a brief statistical analysis, using paired $t$ test to compare means of baseline and follow-up platelet counts and bilirubin and albumin levels. A $P$ value below 0.05 was considered statistically significant. The analysis was performed using IBM statistical product and service solutions statistics for Macintosh, version 21.0 (Armonk, NY, IBM Corp).

\section{RESULTS}

\section{Baseline characteristics of patients}

We analyzed data from 90 consecutive patients with compensated HCV-related cirrhosis who were treated with direct-acting antivirals between January and October 2015 and completed a follow up of at least one year after initiation of therapy.

The median follow-up time after initiation of direct-acting antiviral treatment was 16 months (12-21 months). Seventy-three percent of participants were males, the mean age was 58.1 years and the most frequent genotype was $1 \mathrm{~b}(52.2 \%)$. Only $37.8 \%$ of patients were naïve, and $11 \%$ had liver graft cirrhosis. All patients were Child-Pugh A class at the start of the treatment and the median MELD score was 7 (6-16). At the initiation of therapy, mean bilirubin level was $1.06 \pm 0.27 \mathrm{mg} / \mathrm{dL}$, mean platelet count was $117,788 \pm 50,546 / \mathrm{mm}^{3}$, and mean albumin level was $4,140 \pm 424 \mathrm{mg} / \mathrm{dL}$. The baseline characteristics of the study population are shown in Table 1. 
In terms of treatment, the most frequent combination was sofosbuvir/ledipasvir (35.5\%) and $66.7 \%$ of patients also received ribavirin. Most participants $(78.9 \%)$ were treated for 12 weeks. Six patients $(6.7 \%)$ had relevant adverse effects: 3 patients treated with ribavirin developed moderate anaemia, which improved after lowering the dose; 1 patient referred severe asthenia; 1 patient developed a purpura which required corticosteroids and 1 patient suffered an acute on chronic liver failure which required discontinuation of therapy during the third week of treatment. Eightysix out of 90 patients (94.6\%) achieved SVR12. One patient died during the follow-up due to a metastatic hepatocellular carcinoma. There were no deaths due to unrelated liver causes.

Five patients had a history of hepatocellular carcinoma: 2 patients had one nodule smaller than $5 \mathrm{~cm}, 2$ patients had 3 nodules smaller than $3 \mathrm{~cm}$, and 1 patient had 2 nodules smaller than $2 \mathrm{~cm}$. Three of them underwent liver transplantation between 2004 and 2013 and developed graft cirrhosis. The other 2 patients were treated first with direct-acting antivirals and underwent liver transplantation during the follow-up period.

\section{Hepatocellular carcinoma}

Five patients (5.5\%) developed hepatocellular

Table 1: Baseline characteristics of the study population

\begin{tabular}{lc}
\hline Characteristic & Data, $\boldsymbol{n}(\%)$ \\
\hline Follow-up (months), median (range) & $16(12-21)$ \\
Age (years), mean \pm SD & $58 \pm 8.57$ \\
Gender: male & $66(73.3)$ \\
Treatment & \\
$\quad$ Naive & $34(37.8)$ \\
$\quad$ Experienced & $56(62.2)$ \\
Liver graft cirrhosis & $10(11.1)$ \\
Genotype & \\
1a & $27(30)$ \\
1b & $47(52.2)$ \\
2 & $1(1.1)$ \\
3 & $11(12.2)$ \\
4 & $4(4.5)$ \\
Treatment & \\
Sofosbuvir/ledipasvir & $32(35.5)$ \\
Sofosbuvir/daclatasvir & $24(26.7)$ \\
Sofosbuvir/simeprevir & $16(17.8)$ \\
Paritaprevir/ritonavir/ombitasvir/dasabuvir & $11(12.2)$ \\
Simeprevir/interferon & $4(4.4)$ \\
Sofosbuvir/interferon & $1(1.1)$ \\
Rivabirin: yes & $60(66.7)$ \\
Weeks of treatment & \\
2 & $1(1.1)$ \\
12 & $71(78.9)$ \\
24 & $18(20)$ \\
MELD score, median (range) & $7(6-16)$ \\
History of previous hepatocellular carcinoma & $5(5.6)$ \\
Albumin (mg/dL), mean \pm SD & $4,140 \pm 424$ \\
Platelets (mm ${ }^{3}$ ), mean \pm SD & $117,788 \pm 50,546$ \\
Bilirrubin (mg/dL), mean \pm SD & $1.06 \pm 0.27$ \\
\hline
\end{tabular}

carcinoma [Figure 1]. In one case, a suspicious lesion was detected before treatment and, during the followup, 2 nodules were confirmed with a triple-phase computerized scan, one of $4.8 \mathrm{~cm}$ and one of $1.5 \mathrm{~cm}$, compatible with hepatocellular carcinoma. Another patient had a post-transplant recurrence in the form of lymphatic metastasis 15 months after initiation of therapy (without hepatocellular carcinoma in the liver graft). One patient developed a 4-cm nodule with portal vein thrombosis, one patient had a $3.3-\mathrm{cm}$ nodule and the last patient developed multiple hepatic nodules and bone metastasis. Thus, 3 patients out of 84 (3.6\%) developed de novo hepatocellular carcinoma. The median time between initiation of treatment and the diagnosis of liver cancer was 12 months.

\section{Clinical decompensations}

Four patients $(4.4 \%)$ suffered an episode of hepatic decompensation during the year of follow-up [Figure 2]: 1 patient with non-malignant portal thrombosis developed ascites, 1 patient with a history of ascites developed an acute on chronic liver failure during the treatment, and 2 patients developed ascites coinciding with the diagnosis of hepatocellular carcinoma.

\section{Evolution of liver function}

Seven patients (7.8\%) improved MELD score more than one point, 63 patients $(70 \%)$ showed no differences or \pm one point and in 11 patients (12.2\%) MELD score worsened more than one point. Two patients $(2.2 \%)$ underwent liver transplantation during the follow-up and there was insufficient data to calculate MELD score in 7 patients (7.8\%).

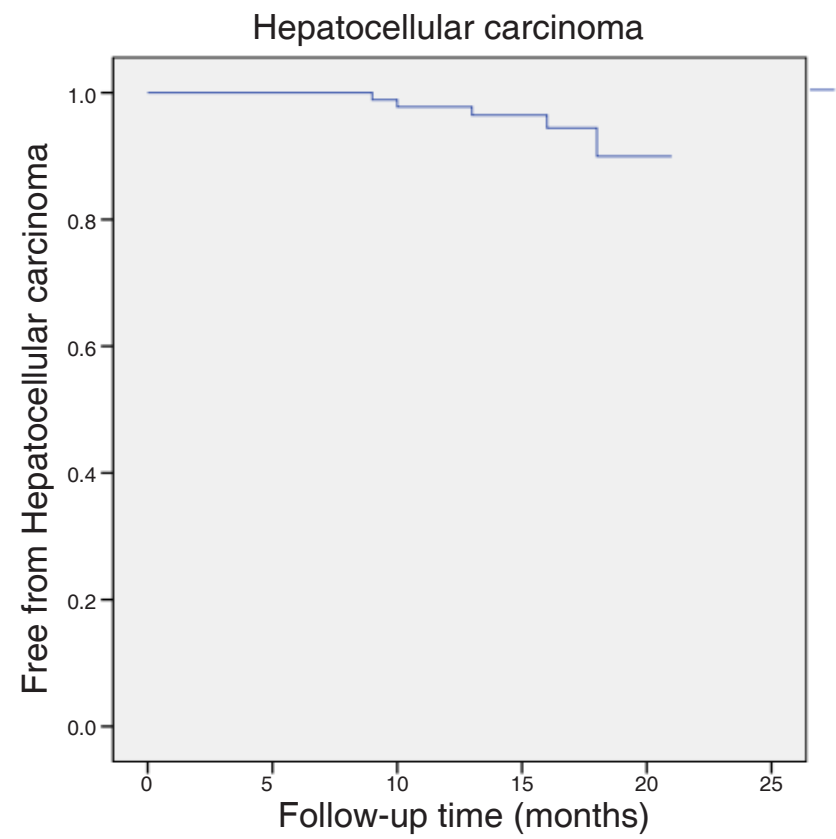

Figure 1: Kaplan Meier estimates of staying free of hepatocellular carcinoma after direct-acting antiviral treatment 


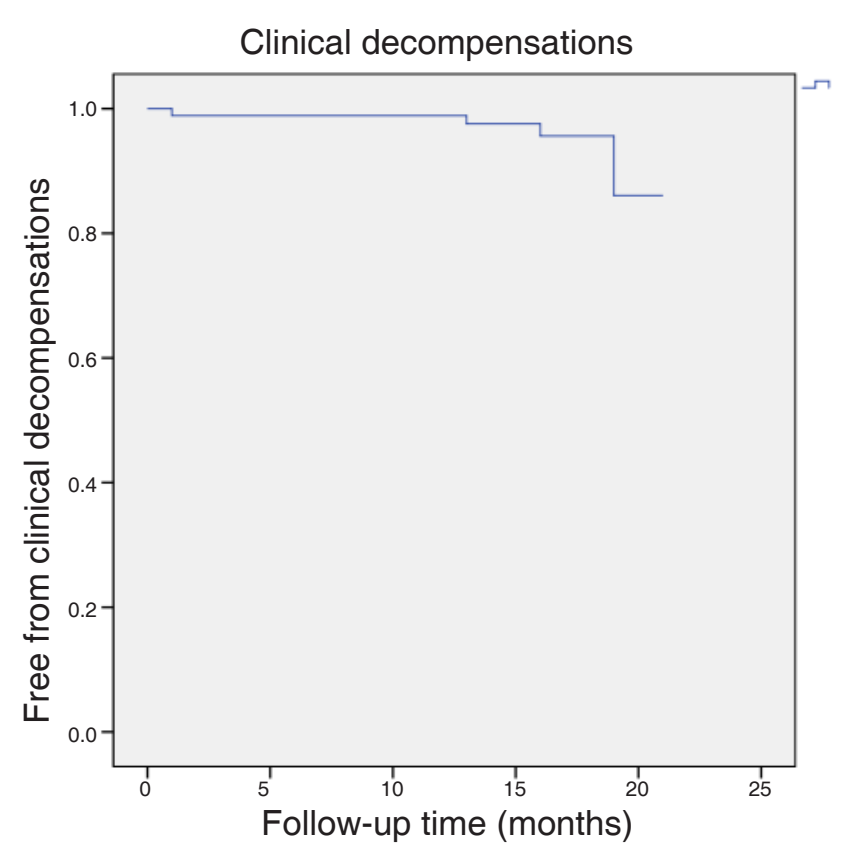

Figure 2: Kaplan Meier estimates of staying free of clinical decompensation after direct-acting antiviral treatment

Seventy-eight patients $(86.7 \%)$ stayed in stage $A$ of Child-Pugh score at the end of the follow-up and 3 patients $(3.3 \%)$ worsened to Child-Pugh B class. Two patients $(2.2 \%)$ underwent liver transplantation during the follow-up and there was insufficient data to calculate Child-Pugh stage in 7 patients (7.8\%).

The statistical analysis showed a significant improvement in the mean platelet counts $(P<0.001$, $95 \% \mathrm{Cl}:-26,360,-12,096)$ and in the mean albumin levels $(P<0.001,95 \% \mathrm{Cl}:-322,-130)$ after antiviral treatment but not in the mean bilirubin level $(P=0.74$, 95\% Cl: $-0.70,0.97)$.

\section{DISCUSSION}

It has been described that patients with advanced chronic liver disease who achieved sustained virological response with interferon-based treatments have a hepatocellular carcinoma annual rate as low as $1 \%(6)$, while for untreated patients it is around $3 \%{ }^{[5,6]}$. In terms of hepatic decompensation, it has been described an annual rate of $1.4 \%$ for patients treated with interferon, in opposition to a $5.7 \%$ for untreated cirrhotic patients ${ }^{[6]}$. Although it is known that direct-acting antiviral therapy has changed the history of chronic HCV infection, achieving very high cure rates and an excellent safety profile ${ }^{[12]}$, a recent prospective study has shown that the resolution of the infection with this treatment in cirrhotic patients does not seem to reduce the incidence of hepatocellular carcinoma in 24 weeks of follow-up ${ }^{[14]}$. Also, a recent publication has suggested that there is a higher risk of tumor recurrence in patients with hepatocellular carcinoma treated with direct-acting antivirals ${ }^{[15]}$, while this association was not found in another prospective study ${ }^{[16]}$. It is important to continue investigating this relationship, developing long-term follow-up studies, in order to clarify the effect that direct-acting antivirals may have on the development of hepatocellular carcinoma and clinical decompensations so that we can better understand and explain to our patients what they can expect after achieving sustained virological response.

In our study, the development of hepatocellular carcinoma in a median of 16 months of follow-up is $3.6 \%$, as high as what it is expected for untreated cirrhotic patients ${ }^{[4,5]}$. Thus, there seems to be no benefits in the short term in this aspect. Undoubtedly, the study has many limitations as it is retrospective, it was performed in a single center, and it has a relatively small sample size. However, not much data have been published to date and the follow-up period is longer than in previous publications.

It is known that the risk of hepatocellular carcinoma increases in advanced stage of fibrosis, in patients with comorbidities such as diabetes mellitus, and in older age ${ }^{[17,18]}$. The reason why there are different outcomes in terms of cancer development depending on the treatment received could be explained by the fact that patients with more advanced liver disease and with comorbidities are now being treated with direct acting antivirals, as they were not considered suitable for interferon based therapies before due to the possibility of dangerous side effects. However, in our study the mean age of patients was only 58-year-old, so we cannot justify the high incidence of hepatocellular carcinoma as a result of the old age of patients treated with direct-acting antivirals. On the other hand, it has been described that alpha-interferon can activate natural killer cells, which are part of innate immunity and play a role in the control of viral infections and tumors ${ }^{[19]}$, while interferon-free regimes produce a rapid decrease of HCV RNA levels which is followed by a rapid decrease in natural killer cells activation ${ }^{[20]}$. This hypothesis could explain the different outcomes between patients receiving one or the other treatment.

In any case, neither direct-acting antivirals nor interferon-based treatments eliminate the risk of hepatocellular carcinoma and patients should continue screening every 6 months after the achievement of sustained virological response.

In terms of clinical decompensation, we also did 
not see a clinical benefit as our study showed a similar risk than what it is expected for untreated cirrhotic patients ${ }^{[4,6]}$. In a recent study it has been found that hepatic venous pressure gradient (HVPG) decreaseed after interferon-free treatment in patients with HCV-related cirrhosis, but in patients with a pretreatment HVPG of $10-15 \mathrm{mmHg}$, clinically significant portal hypertension was only decreased in $43 \%{ }^{[21]}$. In our study, 4 out of 4 patients who developed clinical decompensation had a previous history of ascitis or were diagnosed with hepatocellular carcinoma during the follow-up, suggesting a more advanced disease. This may explain why no clinical benefit was observed in these patients, and suggests that a longer follow-up period may be needed.

In terms of the hepatic function, our study did not show a significant improvement in the Child-Pugh and MELD scores. This may be because pre-treatment scores were already low. We also had no control group, making it difficult to determine if the outcomes were better or worse than expected. We therefore performed a statistic analysis comparing the mean platelet counts and bilirubin and albumin levels pre- and posttreatment which reflects the hepatic function. We found that there is a statistically significant improvement in platelet counts and in albumin levels showing some benefit in direct-acting antiviral treatment in the short term.

In conclusion, direct-acting antiviral treatment is not associated with a decrease in the development of hepatic decompensations or hepatocellular carcinoma in the first year of follow-up compared to literature reports for untreated compensated cirrhotic patients. On the other hand, there is a statistically significant improvement in platelet counts and in albumin levels, showing a possible improvement of the hepatic function in the short term. More studies are needed to determine the benefits of direct-acting antiviral therapy in the long term.

\section{DECLARATIONS}

\section{Acknowledgments}

We thank Dr. Mei-Sze Chua for language polish of this manuscript.

\section{Authors' contributions}

Concept and design: E. Berge, A. Arencibia, F. Pérez Data acquisition: E. Berge, A. Arencibia, L. Cejas, S. Acosta

Data analysis: E. Berge, F. Pérez

Manuscript preparation: E. Berge, E. Otón, L. Cejas, S. Acosta
Critical revision: A. Arencibia, E. Otón, F. Pérez

\section{Financial support and sponsorship}

None.

\section{Conflicts of interest}

E. Berge, A. Arencibia, E. Otón, L. Cejas and S. Acosta declare that they do not have anything to disclose with respect to this manuscript. F. Pérez: Advisory board for Abbvie, BMS, Gilead, Janssen and MSD.

\section{Patient consent}

The data obtained through the medical record review were managed according to the privacy policy and ethics code of our institute.

\section{Ethics approval}

This was a retrospective study and did not require Institutional Review Board approval.

\section{REFERENCES}

1. Lavanchy D. Evolving epidemiology of hepatitis C virus. Clin Microbiol Infect 2011;17:107-15.

2. Thein HH, Yi Q, Dore GJ, Krahn MD. Estimation of stage-specific fibrosis progression rates in chronic hepatitis $\mathrm{C}$ virus infection: a meta-analysis and meta-regression. Hepatology 2008;48:418-31.

3. Westbrook RH, Dusheiko G. Natural history of hepatitis C. J Hepatol 2014;61:S58-68.

4. Fattovich G, Giustina G, Degos F, Tremolada F, Diodati G, Almasio P, Nevens F, Solinas A, Mura D, Brouwer JT, Thomas H, Njapoum C, Casarin C, Bonetti P, Fuschi P, Basho J, Tocco A, Bhalla A, Galassini R, Noventa F, Schalm SW, Realdi G. Morbidity and mortality in compensated cirrhosis type C: a retrospective follow-up study of 384 patients. Gastroenterology 1997;112:463-72.

5. Sangiovanni A, Del Ninno E, Fasani P, De Fazio C, Ronchi G, Romeo R, Morabito A, De Franchis R, Colombo M. Increased survival of cirrhotic patients with a hepatocellular carcinoma detected during surveillance. Gastroenterology 2004;126:1005-14.

6. Fattovich G, Giustina G, Degos F, Diodati G, Tremolada F, Nevens F, Almasio P, Solinas A, Brouwer JT, Thomas H, Realdi G, Corrocher $\mathrm{R}$, Schalm SW. Effectiveness of interferon alfa on incidence of hepatocellular carcinoma and decompensation in cirrhosis type C. European Concerted Action on Viral Hepatitis (EUROHEP). J Hepatol 1997;27:201-5.

7. Bota S, Sporea I, Popescu A, Sirli R, Neghina AM, Danila M, Strain M. Response to standard of care antiviral treatment in patients with HCV liver cirrhosis - a systematic review. J Gastrointestin Liver Dis 2011;20:293-8.

8. Ward RP, Kugelmas M. Using pegylated interferon and ribavirin to treat patients with chronic hepatitis C. Am Fam Physician 2005;72:655-62.

9. Singal AK, Singh A, Jaganmohan S, Guturu P, Mummadi R, Kuo YF, Sood GK. Antiviral therapy reduces risk of hepatocellular carcinoma in patients with hepatitis C virus-related cirrhosis. Clin Gastroenterol Hepatol 2010;8:192-9.

10. Singal AG, Volk ML, Jensen D, Di Bisceglie AM, Schoenfeld PS. A sustained viral response is associated with reduced liver-related 
morbidity and mortality in patients with hepatitis $\mathrm{C}$ virus. Clin Gastroenterol Hepatol 2010;8:280-8.

11. van der Meer AJ, Berenguer M. Reversion of disease manifestations after HCV eradication. J Hepatol 2016;65:S95-108.

12. Nakamoto S, Kanda T, Shirasawa H, Yokosuka O. Antiviral therapies for chronic hepatitis $\mathrm{C}$ virus infection with cirrhosis. World J Hepatol 2015;7:1133-41.

13. Horsley-Silva JL, Vargas HE. New therapies for hepatitis C virus infection. Gastroenterol Hepatol (N Y) 2017;13:22-31.

14. Conti F, Buonfiglioli F, Scuteri A, Crespi C, Bolondi L, Caraceni P, Foschi FG, Lenzi M, Mazzella G, Verucchi G, Andreone P, Brillanti $\mathrm{S}$. Early occurrence and recurrence of hepatocellular carcinoma in HCV-related cirrhosis treated with direct-acting antivirals. $J$ Hepatol 2016;65:727-33.

15. Reig $M$, Mariño Z, Perelló $C$, Iñarrairaegui $M$, Ribeiro $A$, Lens $S$, Díaz A, Vilana R, Darnell A, Varela M, Sangro B, Calleja JL, Forns $\mathrm{X}$, Bruix J. Unexpected high rate of early tumor recurrence in patients with HCV-related HCC undergoing interferon-free therapy. $J$ Hepatol 2016;65:719-26.

16. ANRS collaborative study group on hepatocellular carcinoma. Lack of evidence of an effect of direct-acting antivirals on the recurrence of hepatocellular carcinoma: data from three ANRS cohorts. $J$ Hepatol 2016;65:734-40

17. Balogh J, Victor D 3rd, Asham EH, Burroughs SG, Boktour M Saharia A, Li X, Ghobrial RM, Monsour HP Jr. Hepatocellular carcinoma: a review. J Hepatocell Carcinoma 2016;3:41-53.

18. Baumert TF, Jühling F, Ono A, Hoshida Y. Hepatitis C-related hepatocellular carcinoma in the era of new generation antivirals. $B M C$ Med 2017;15:52.

19. Yoon JC, Yang CM, Song Y, Lee JM. Natural killer cells in hepatitis C: current progress. World J Gastroenterol 2016;22:1449-60.

20. Tatsumi $\mathrm{T}$, Takehara $\mathrm{T}$. Impact of natural killer cells on chronic hepatitis $\mathrm{C}$ and hepatocellular carcinoma. Hepatol Res 2016;46:416-22.

21. Mandorfer M, Kozbial K, Schwabl P, Freissmuth C, Schwarze R, Stern R, Chromy D, Stättermayer AF, Reiberger T, Beinhardt S, Sieghart W, Trauner M, Hofer H, Ferlitsch A, Ferenci P, PeckRadosavljevic M. Sustained virologic response to interferon-free therapies ameliorates $\mathrm{HCV}$-induced portal hypertension. J Hepatol 2016;65:692-9. 